\title{
BMJ Open Predicting the risk of cancer in adults using supervised machine learning: a scoping review
}

\author{
Asma Abdullah Alfayez (D) , ${ }^{1,2}$ Holger Kunz (D) , ${ }^{1}$ Alvina Grace Lai (D) ${ }^{1}$
}

To cite: Abdullah Alfayez A, Kunz H, Grace Lai A. Predicting the risk of cancer in adults using supervised machine learning: a scoping review. BMJ Open 2021;11:e047755. doi:10.1136/ bmjopen-2020-047755

- Prepublication history for this paper is available online. To view these files, please visit the journal online (http://dx.doi. org/10.1136/bmjopen-2020047755).

Received 20 December 2020 Accepted 01 September 2021

\section{Check for updates}

(C) Author(s) (or their employer(s)) 2021. Re-use permitted under CC BY. Published by BMJ.

${ }^{1}$ Institute of Health Informatics, University College London, London, UK

${ }^{2}$ King Abdullah International Medical Research Center, Riyadh, Saudi Arabia

Correspondence to Ms Asma Abdullah Alfayez; asma.alfayez.17@ucl.ac.uk and Dr Alvina Grace Lai; alvina.lai@ucl.ac.uk

\section{ABSTRACT}

Objectives The purpose of this scoping review is to: (1) identify existing supervised machine learning (ML) approaches on the prediction of cancer in asymptomatic adults; (2) to compare the performance of ML models with each other and (3) to identify potential gaps in research. Design Scoping review using the population, concept and context approach.

Search strategy PubMed search engine was used from inception to 10 November 2020 to identify literature meeting following inclusion criteria: (1) a general adult ( $\geq 18$ years) population, either sex, asymptomatic (population); (2) any study using ML techniques to derive predictive models for future cancer risk using clinical and/ or demographic and/or basic laboratory data (concept) and (3) original research articles conducted in all settings in any region of the world (context).

Results The search returned 627 unique articles, of which 580 articles were excluded because they did not meet the inclusion criteria, were duplicates or were related to benign neoplasm. Full-text reviews were conducted for 47 articles and a final set of 10 articles were included in this scoping review. These 10 very heterogeneous studies used ML to predict future cancer risk in asymptomatic individuals. All studies reported area under the receiver operating characteristics curve (AUC) values as metrics of model performance, but no study reported measures of model calibration.

Conclusions Research gaps that must be addressed in order to deliver validated ML-based models to assist clinical decision-making include: (1) establishing model generalisability through validation in independent cohorts, including those from low-income and middle-income countries; (2) establishing models for all cancer types; (3) thorough comparisons of ML models with best available clinical tools to ensure transparency of their potential clinical utility; (4) reporting of model calibration performance and (5) comparisons of different methods on the same cohort to reveal important information about model generalisability and performance.

\section{INTRODUCTION}

Cancer remains a leading cause of morbidity and mortality, with an estimated 1.8 million new cases and 0.6 million deaths in the USA in 2019 and approximately 367000 new cases and 165000 cancer deaths in the UK each year between 2015 and $2017 .{ }^{12}$ Annual death rates

\section{Strengths and limitations of this study}

This study used a recognised scoping review approach (population, concept and context) to explore the machine learning techniques used to derive predictive models for future cancer risk.

- Identified studies were not subjected to comprehensive qualitative assessments.

- Only 10 studies were identified, making it difficult to draw firm conclusions about their relative performance.

- Area under the curve values (AUC) alone do not allow for meaningful comparisons of models as they have been trained and evaluated on different datasets under different circumstances and conditions.

- This scoping review is limited to papers published in English until 2020 and only the PubMed search engine was used.

only modestly decreased $(1.4 \%$ and $1.8 \%$ in women and men, respectively) between 2012 and 2016, despite significant research. ${ }^{1}$ Cancer cases also continue to increase, not least due to increased life expectancy, which increases the risk of developing cancer. ${ }^{3}$

Early cancer diagnosis is associated with significantly higher survival rate and lower mortality and associated costs. Early-stage cancers require less complex treatment regimens and reduced hospital utilisation, resulting in reduced healthcare costs, whereas late-stage cancers require complex multimodal management, several rounds of extremely expensive drugs over significant periods of time, and the treatment of recurrences, equating to a staggering economic burden. Therefore, the importance of early diagnosis cannot be overestimated. ${ }^{4-6}$ Treating cancer early has significant costsaving benefits. In the USA, during the first 24 months after diagnosis, there is an increase in cancer treatment costs with stage: US\$72000 for stage 0, US\$97000 for stage I/ II, US $\$ 159000$ for stage III and US $\$ 182000$ for stage IV. ${ }^{7}$ An estimate of the cost savings from early cancer diagnosis is US $\$ 26$ billion 
per annum in the USA alone. ${ }^{8}$ Similarly, in the UK, early diagnosis of colorectal, ovarian, and lung cancer in England alone could provide savings of over $£ 44$ million and benefit nearly 11000 patients. $^{9}$

Survival rates significantly improve if cancer is diagnosed at stage I or II compared with later stages (stage III and IV),${ }^{10} 11$ as once the cancer has metastasised, it becomes difficult to treat with radiotherapy or surgery, leading to treatment failure and death. For example, 5 -year survival rates for women diagnosed with localised breast or ovarian cancer are 99\% and 92\% compared with $27 \%$ and $29 \%$ for metastatic disease, respectively. ${ }^{1}$ A report by Cancer Research UK indicated that, in the UK, the 10-year survival proportions of patients with eight cancers (combined) were around $80 \%$ for stage I and stage II detection (breast, bladder, ovarian, colorectal, uterine, testicular, cervical, and malignant melanoma) but only $26 \%$ for cancers detected at later stages, notably lung cancer (stage III and IV). ${ }^{12}$

\section{Current approaches to diagnose incident cancer}

One approach to the early detection of cancer is population-wide screening, which aims to find asymptomatic individuals so that they can be promptly referred for treatment. Examples include mammography for breast cancer, cervical screening for cervical cancer and faecal occult blood testing or sigmoidoscopy for colorectal cancer. ${ }^{13}$ There are three examples of national screening programmes in UK (bowel, breast and cervical cancer screening programs ${ }^{14}$ and two in the USA: the Colorectal Cancer Control Programme (CRCCP) and the National Breast and Cervical Cancer Early Detection Programme (NBCCEDP).${ }^{15}$ However, significant proportions of individuals eligible for these programmes do not participate (eg, through fear or not prioritising time to attend for screening),${ }^{16}$ and comprehensive screening programmes are costly to implement, especially in resource-poor settings or low-income and middle-income countries. Other approaches include public health campaigns to encourage individuals experiencing particular symptoms such as weight loss, anorexia and fatigue to visit their family doctors. ${ }^{17}$ However, patient help-seeking around cancer is complex, multistaged, and often leads to long delays of weeks or even months. ${ }^{18}$ Patients find it hard to interpret and recognise symptoms, with fears of embarrassment and having a potentially fatal or painful condition contributing to long and avoidable delays in help-seeking from health professionals. ${ }^{18}{ }^{19}$ Patients often do not seek help from health professionals for early cancer symptoms, notably from general family physicians, for many reasons including a complex mix of fear, worry and of 'wasting' health professionals' time ${ }^{19}$ or due to the high costs of medical care, a lack of health insurance or time constraints. ${ }^{20}$

\section{Detecting future risk of cancer by modelling data}

Screening approaches represent a patient identification (or 'phenotyping' problem) that aims to detect whether the individual has cancer at a particular point in time. However, the ultimate goal of cancer prediction is to determine whether an individual will develop cancer at some point in the future. A simple approach is to stratify populations according to the presence and absence of risk factors, which have been extensively characterised for most cancer types through epidemiological studies over many decades. For example, age, gender, ethnicity, family history and lifestyle factors are well-established risk factors for many types of cancer. ${ }^{21}$ The cancer prediction problem can either be regarded as a regression problem, where the input variables are clinical-demographic variables and the output variable is the probability of developing cancer at some point in the future, or as a binary classification problem to determine whether or not a patient will develop cancer at a specific point in time.

\section{Big data and machine learning for medical prediction models}

Advances in digital medicine and computational science have altered the landscape of data available for cancer risk prediction models. For example, in the data-driven healthcare era, there is an increasing amount of 'big' medical data, as most individuals have had interactions with the healthcare system where data is collected in the form of electronic health records (EHRs), which are systematic collections of longitudinal patient health data collected in real time. ${ }^{22}{ }^{23}$ Such large datasets provide powerful new opportunities to develop and refine predictive models and to explore potentially unknown predictor variables. ${ }^{22}$ Leveraging often massive amounts of data generated from large populations, much of which may be unstructured, and building optimal models requires the exploitation of advanced computational tools and supporting infrastructure. Machine learning (ML) is a branch of artificial intelligence and an extension of traditional statistical techniques that uses computational resources to detect underlying patterns in high-dimensional data, and it is increasingly being used in different areas of medicine requiring predictions. ${ }^{24}$ For example, ML has successfully been used with EHR data to predict incident hypertension ${ }^{25}$ and incident chronic kidney disease,${ }^{26}$ and wider popular uses of ML in medicine include the automatic interpretation of medical images such as in radiology ${ }^{27}$ and histopathology ${ }^{28}$ images.

\section{A brief description of ML}

A comprehensive description of ML models is beyond the scope of this scoping review. However, relevant ML techniques relate to the problem of learning from data samples (eg, EHR data) rather than being preprogrammed with existing knowledge or rules. ML models can either be supervised (ie, where the data are labelled and the algorithm uses these data to learn to predict the output) or unsupervised (ie, where the data are unlabelled and the algorithm learns a structure inherent in the data). ${ }^{29}$ The cancer prediction problem is, therefore, a supervised problem; examples are provided as inputs (or features) such as cancer risk factors like age, history, ethnicity or 
blood count parameters and outputs (or labels) such as whether or not the individual subsequently develops cancer. A variety of available algorithms learn the best way to map the features to the labels by learning from the observations. ${ }^{3031}$ The resulting model, ideally, will then be able to generalise the information so that it can be applied with high precision to new and unseen data. ${ }^{30} 31$

Some of the main supervised ML models used in medical applications include decision trees (DTs; and their adaptation, random forests (RFs)), support vector machines (SVMs) and artificial neural networks (ANNs). ${ }^{30}{ }^{31} \mathrm{DTs}$ produce an output similar to a flow chart formed from feature nodes (risk variables) that best discriminate between different labels (future cancer occurrence) to split the tree. ${ }^{3031}$ In this way, new cases can be assessed by traversing the tree based on the feature values to determine the output for that example. ${ }^{30}{ }^{31}$ DTs are easy to interpret, since users are usually able to visualise the steps leading to a particular classification, which may be useful in a clinical setting where experts might wish to see how a particular decision was made. ${ }^{30}{ }^{31} \mathrm{In}$ RFs, several trees are built using subsets of data and features, with predictions decided based on majority voting after the example is assessed with respect to all the constructed trees. ${ }^{30} 31$

In SVMs, each feature (risk factor) is mapped into a higher-dimensional space and the hyperplane that optimally separates the output (future cancer occurrence) modelled. ${ }^{30}{ }^{31}$ SVMs tend to generalise well to unseen data and work well with complex (multidimensional) data but can be hard to interpret. ${ }^{3031}$

ANNs are inspired by the neural connections in the human brain and are developed by creating nodes (neurons) that weight certain features and produce an output value. ${ }^{30} 31$ By layering nodes in between the input layer (features; cancer risk factors) and output layer (label; future cancer occurrence) and modifying the weights during learning through a process called backpropagation, the resulting model forms a prediction for unseen data when one of the nodes in the output layer is positive. ${ }^{30}{ }^{31}$ The terms 'deep neural network' and 'deep learning' are applied to ANNs with large numbers of layers. ${ }^{30}{ }^{31}$ While proving extremely powerful across a range of applications, ANNs can be computationally very expensive and the way in which they classify (ie, the intermediate 'hidden' layers) is opaque, making it difficult to determine exactly how they performed the classification problem. ${ }^{30} 31$

\section{Rationale for performing a scoping review}

ML remains a relatively recent field, so it is unclear exactly to what extent advances have impacted specific healthcare domains. There are currently no extended systematic reviews or scoping reviews on the application of ML to cancer risk prediction in asymptomatic individuals. This prompted us to perform a scoping review of studies using supervised ML techniques to predict the future risk of developing cancer or specific cancers within a general asymptomatic adult ( $\geq 18$ years) population using clinical and/or demographic and/or basic laboratory data (eg, complete blood counts (CBC)) that are likely to be readily available within the primary care setting. This approach, therefore, allowed to: (1) identify the types of evidence available; (2) clarify key concepts and definitions; (3) examine how research is currently being conducted and (4) to identify knowledge gaps. ${ }^{32}$

\section{Objectives}

The objective of this study was to perform a scoping review and to synthesise knowledge of the nature and effects of current ML techniques for early cancer detection in asymptomatic adults. The scoping review was guided by the following research questions:

1. Which, if any, ML methods are being developed for cancer risk prediction in asymptomatic individuals in the community?

2. How do these models perform compare to each other?

3 . Which research or knowledge gaps need to be addressed in order to advance the field?

\section{METHODS}

\section{Inclusion and exclusion criteria}

We used the population, concept and context approach ${ }^{33}$ with the following inclusion criteria: (1) general adult ( $\geq 18$ years) population, either sex, asymptomatic (population); (2) any study using ML techniques to derive predictive models for future cancer risk using clinical and/or demographic and/or basic laboratory data carried out prior to 7 August 2020 (concept) and (3) original research articles conducted in all settings in any region of the world (context).

For the purposes of this study, and recognising that 'ML' algorithms fall along a continuum with statistical techniques, ${ }^{34}$ all modelling approaches were included were defined as ML in the respective papers (such as logistic regression (LR)). Exclusion criteria were any ML model used to predict future events in patients with pre-existing or symptoms of cancer; ML models developed using specialised tests such as genetic profiling or imaging tests not generally available in the community; unsupervised ML models; and studies not written in English.

\section{Literature search}

To identify relevant studies, the PubMed database was searched from inception through to November 10, 2020 using the search string: "Cancer" Or "Cancers" OR "Oncology") AND ("Machine Learning" OR "ML" OR "Data Mining" OR "Decision Support System" OR "Clinical Support System" OR "Classification" OR "Regression" OR "Support vector machines" OR "Gaussian process" OR "Neural networks" OR "Logical learning" OR "Bayesian network" OR "linear model") AND ("prognosis" OR "prognostic estimate" OR "predictor" OR "prediction" OR "model" OR "diagnosis" OR "diagnostic"). This search was supplemented with manual searching of the references and citations of previously published studies. All abstracts identified by the initial search were screened 
for inclusion and checked for accuracy. For the included studies, data were extracted from full papers. In instances where more information was required to determine inclusion, the full text of the article was retrieved and assessed against the eligibility criteria.

\section{Study assessment}

The quality of the included studies was assessed using the Newcastle Ottawa Scale (NOS) for observational studies included in the review. ${ }^{35}$ The strength of the predictive ability of the included models was assessed using area under the receiver operating characteristics curve (AUC) data, a valid measure for evaluating classification algorithms and one that has been used to compare different algorithms in other meta-analyses. ${ }^{36} 37$

\section{Patient and public involvement}

This study was not explicitly informed by patient priorities, experiences and preferences, although the application of predictive models to assess cancer risk would have a direct bearing on identifying those most at risk and implementing investigations in a timely manner. No patients were involved in the design or conduct of the study and since this was a scoping review of the literature, there were no study participants.

\section{RESULTS \\ Main findings}

Identified risk models

Using the search strategy, 627 initial studies were identified where 10 studies met the inclusion criteria (table 1 and figure 1). ${ }^{31}{ }^{38-47}$ The most common reasons for exclusion of studies were: (1) models were derived to predict outcomes or responses to therapy in patients with pre-existing cancer and/or (2) the studies used features other than clinical and/or demographic and/ or basic laboratory data, such as genetic biomarkers. All studies were retrospective cohort or case-control studies conducted between 2011 and 2020, with 8 out of 10 studies completed in the last 2 years. Eight studies were conducted in the USA and two in Taiwan. One model was built for breast cancer, three for colorectal cancer, one for lung cancer, one for melanoma, two for nonmelanoma skin cancer, one for pancreatic cancer and one a general cancer prediction model. Two studies performed external validations of a previously developed colorectal cancer prediction model (table 1) ${ }^{39} 43$ In terms of quality assessment, four studies were graded as 'good' quality by the NOS, ${ }^{39} 434446$ while six studies were graded as 'poor', in all cases due to comparability of cohorts on the basis of the design or analysis adequately controlling for confounders. ${ }^{313840424547}$

\section{Development of the risk models}

The models developed in the studies employed a wide range of ML techniques. Two studies compared different modelling approaches on the same dataset, ${ }^{41}{ }^{44}$ while the other eight developed a model using a single approach. The following ML approaches were used: ANNs (8 out of 10 studies), LR (2/10 studies), Gaussian naïve Bayes ( 1 out of 10 studies), Bayesian network inference (1/10 studies), DTs (1/10 studies) and RFs (2/10 studies), linear discriminant analysis (LDA) (1/10 studies), and SVMs (1/10 studies) (table 1$)$. Data for training and testing were from medical insurance databases $(4 / 10$ studies), EHR data repositories (3/10 studies), surveys (2/10 studies) or represented a retrospective analysis of prospectively collected data from a clinical trial $(1 / 10$ studies).

As a result of the diverse cancer types being modelled, study aims and the available data, a range of different predictors, features and/or risk factors were included the developed predictive models, which can be grouped into the following categories: (1) patient demographic data, for example, age, gender, ethnicity, family history; (2) social and lifestyle data, for example, cigarette smoking and intensity of exercise; (3) comorbidities, for example, diabetes mellitus, hypertension, congestive heart failure and chronic obstructive pulmonary disease; (4) clinical and practice data, for example, World Health Organisation - Anatomical Therapeutic Chemical (WHO-ATC) prescription codes and clinical encounters and (5) laboratory tests, for example, $\mathrm{CBC}$ (table 1). The models that automatically extracted features from EHR records used features that were not always explicitly defined in the respective articles.

\section{Discrimination and calibration of the risk models}

All studies provided AUC values as an assessment of model performance. Calibration (ie, whether the risk estimates were accurate), was not assessed in any study. Two models with particularly high AUC values were the Bayesian network inference model developed by Zhao et $a t^{47}$, which used 20 demographic, lifestyle, symptom, comorbidity and lab test results to predict the risk of pancreatic cancer with an AUC of 0.91, and the CRC predictive model developed by Wang $e t a t^{46}$, which used a CNN learning on 1929 features (1099 International Classification of Diseases, Ninth Revision (ICD-9) codes and 830 ATC codes). Models with particularly low AUC values were the range of models (LR, Gaussian naive Bayes, DT, LDA, SVM and feed-forward ANN) developed by Stark $e t$ $a l^{44}$; however, as discussed below, although these models only had AUCs between 0.51 and 0.61 , two of the models compared favourably with the BRCAT clinical risk tool.

\section{Comparison of the risk models with existing predictive algorithms}

Hundreds of risk prediction models have been published in the literature for every cancer type, and some of these are already used in clinical practice. It is, therefore important to understand whether the performance of the newer ML-based cancer risk models is comparable to that of existing predictive algorithms. We, therefore, specifically examined whether the studies compared their ML 


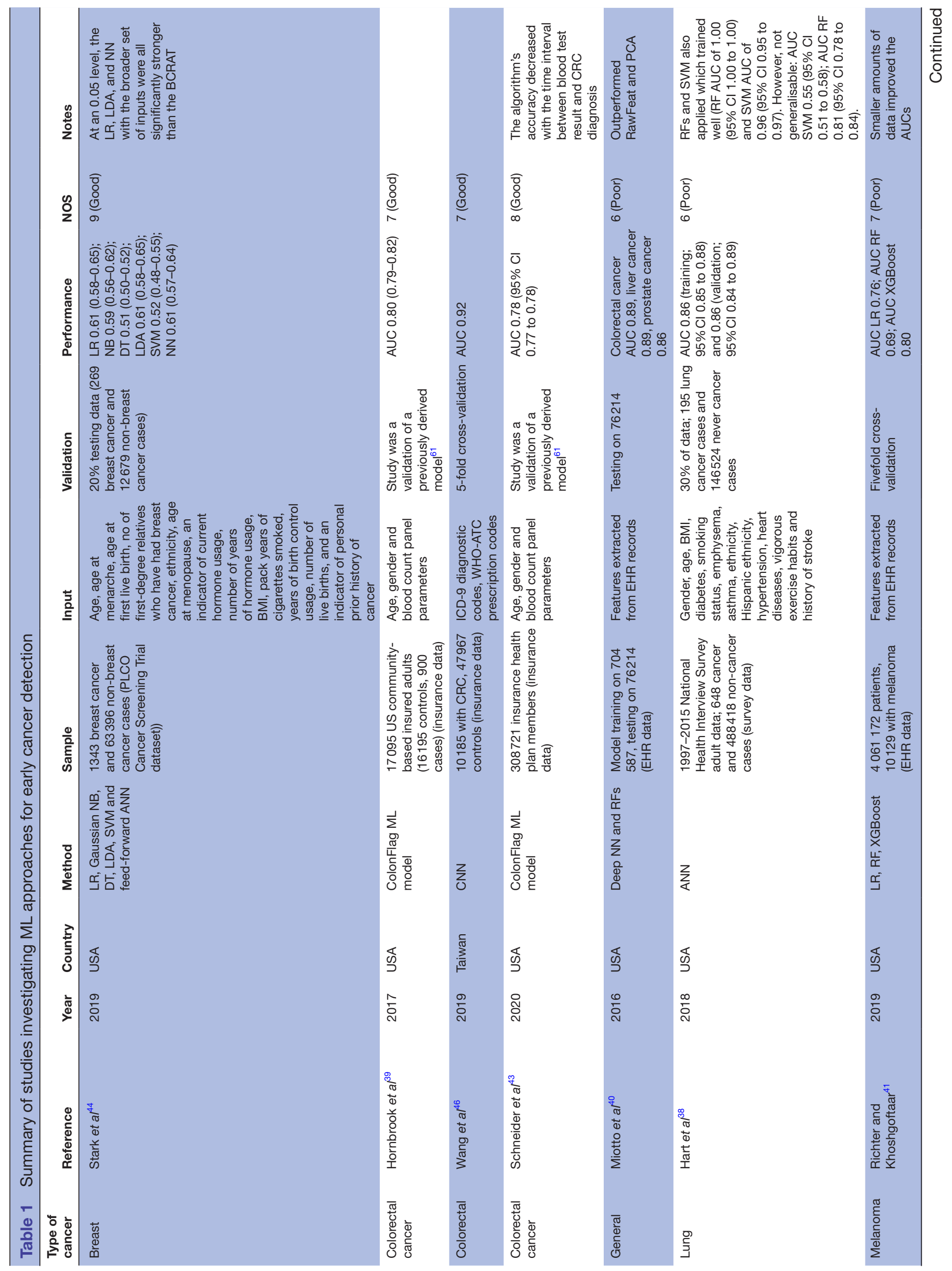




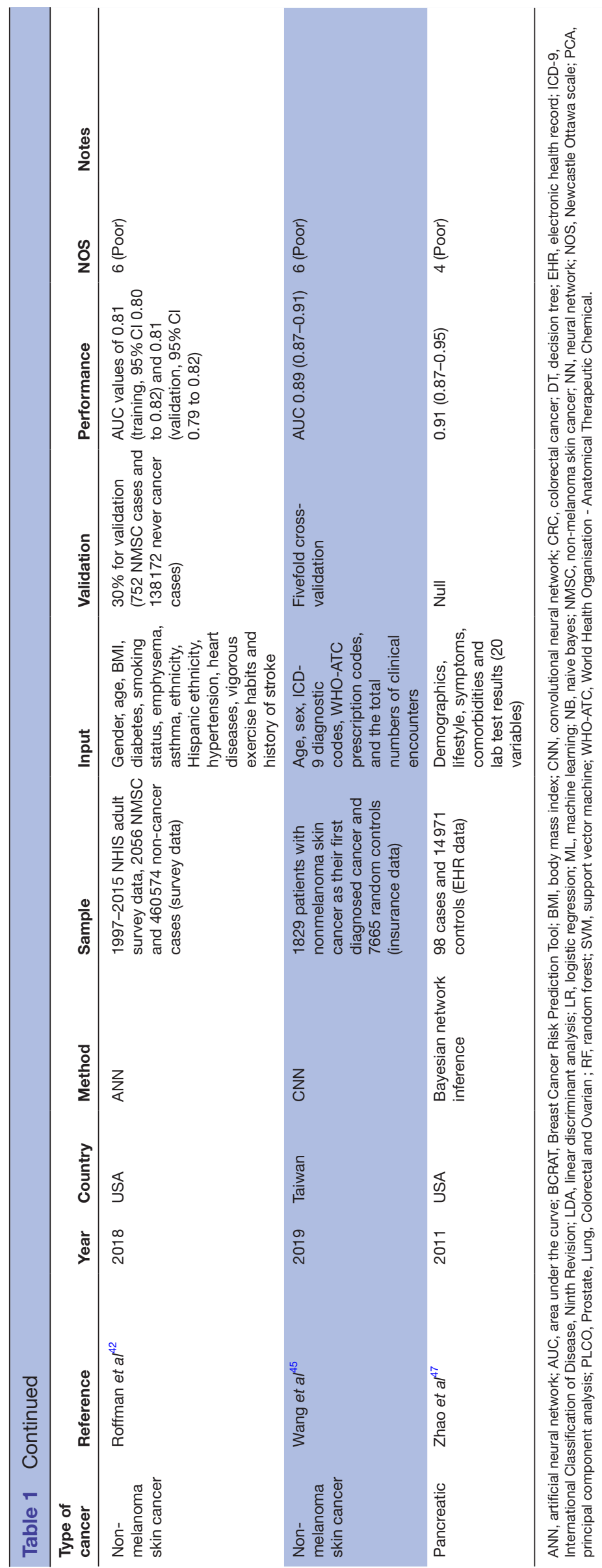

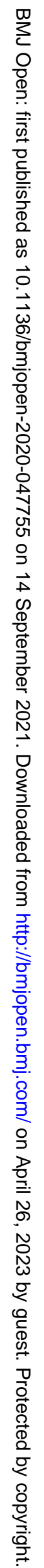




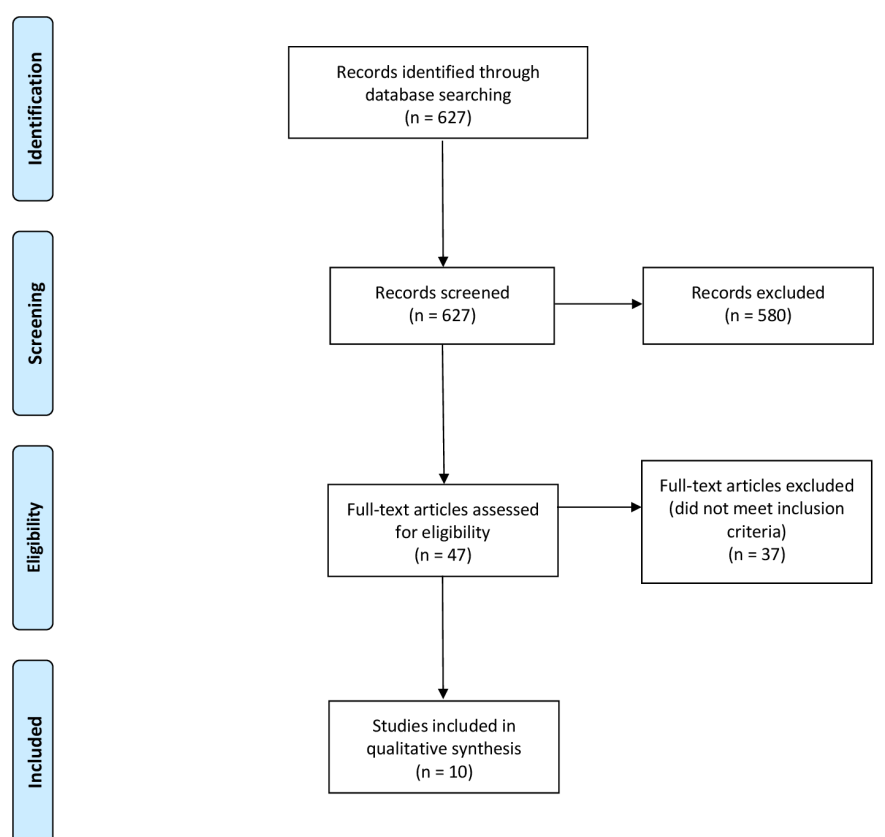

Figure 1 PRISMA flow chart depicting the search strategy. PRISMA, Preferred Reporting Items for Systematic Reviews and Meta-Analyses.

algorithms with existing algorithms or, if not, how model performance as described by AUCs compared with other published data, despite the limitations of this approach (see below).

Stark et $a t^{44}$ compared their ML models with an existing clinical prediction tool, the Breast Cancer Risk Prediction Tool (BCRAT; https://bcrisktool.cancer.gov/). The BCRAT tool is an implementation of the Gail model, ${ }^{48}$ which is a statistical model that estimates 5-year breast cancer risk in women without a personal history of breast cancer and without known mutations in highrisk breast cancer genes such as BRCA1 and BRCA2. In the Gail model, patients self-report their current age, age at menarche, age at first live birth, number of firstdegree relatives who have had breast cancer, ethnicity, and number of previous breast biopsies, variables which are weighted within the model by LR. ${ }^{48}$ In addition, BCRAT uses data on a personal history of atypical hyperplasia, where available. Although the AUC values for the models (LR, naïve Bayes, DTs, LDA, SVM and an ANN) tested using a broader set of features than BCRAT were only between 0.51 (DT) and 0.61 (LR, LDA, and ANN), four of the six models (LR, NB, LDA and ANN) outperformed BCRAT (AUC 0.56). Other metrics were also used to assess model performance (sensitivity, specificity and precision), which were comparable between the ML algorithms and the BCRAT, and both BCRAT and the ML models had low precision $(\sim 2 \%)$. Furthermore, when comparing the different ML models, LR and LDA produced higher AUCs than the ANN model, despite the potential for ANNs to better model noisy data and complex non-linear functions. ${ }^{49}$ The authors suggested that this might have been due to the limited amount of available training data or the selection of hyperparameters. ${ }^{44}$ It was observed that (1) the derived ML models using an extended and set of features available in primary care can deliver improvements on current clinical algorithms; (2) that adding additional features has a greater impact on improving model performance (ie, higher AUC) rather than simply using more complex models and (3) that AUC values must be interpreted in the context of existing methods, such as existing, clinically used risk prediction models such as the BCRAT or Gail model, rather than in isolation.

In a systematic review of 52 colorectal cancer models predicting future risk of disease in asymptomatic individuals, ${ }^{50} 37$ models reported AUC values, which ranged from 0.65 and 0.75 . These included five models that used routine data exclusively and did not include questionnaires or genetic biomarkers. In comparison, the AUC values for ColonFlag, ${ }^{39} 43$ an ML model that uses age, gender and CBC features to predict the future occurrence of colorectal cancer up to 12 months prior to diagnosis, were $0.78-0.82$.

In another systematic review involving 25 risk prediction models for lung cancer that used only epidemiological parameters as input (ie, no laboratory parameters), ${ }^{51}$ AUCs ranged between 0.57 and 0.86 , which compares to an AUC of 0.86 (in both training and validation cohorts) for the ANN model developed by Hart et al. ${ }^{38}$ In their systematic review of 25 melanoma risk prediction models, Usher-Smith et $a \tilde{l}^{2}$ showed in a summary ROC curve that most models had similar discrimination of 0.76 , which compares to the highest AUC of 0.80 achieved using XGBoost ML by Richter et $a l^{41}$.

\section{DISCUSSION}

\section{Strengths and limitations of existing ML approaches}

The reviewed studies reviewed highlight that several different techniques have successfully been used to develop models and that ML can be applied to large-scale insurance and EHR data containing hundreds or thousands of features in order to build predictive models. However, the survey also highlights a number of gaps in the application of ML to predicting the risk of future cancer in asymptomatic individuals. These can be divided into those relating to: (1) study populations; (2) model types and comparisons and (3) model validation, comparisons and calibration.

\section{Study populations}

To date, ML techniques have only been applied to or validated in datasets from developed countries, representing a fraction of the overall global population and their dietary and lifestyle factors. Given that the aetiology of cancer, risk factors and genetics differ in different populations, ${ }^{53}$ models developed in populations in high-income countries may not be generalisable to those from low- and middle-income countries (LMICs). The development and validation of models in LMICs could have two advantages: 
first, it would determine the generalisability (and therefore utility) of that model in other populations, better serving the needs of individuals in LMICs; second, disparities between models developed in different geographical settings could provide valuable new information about factors contributing to cancer risk. Generalising risk prediction models is likely to be challenging, since resource-poor countries often do not have the necessary infrastructure nor the epidemiological research capabilities of institutions in high-income countries.

Furthermore, current ML models predict the risk of a limited number of cancer types. Although breast, colorectal and lung cancer are the three most common cancers and therefore account for a large proportion of overall cancer burden, it is still important to detect all cancers early. This is especially true for those cancers that are usually silent (asymptomatic) for long periods of time, present late with advanced-stage disease, and for which there are currently no screening programmes in place, such as ovarian and pancreatic cancer. Predicting future risk of these cancers could allow closer monitoring of at-risk individuals.

\section{Model types and comparisons}

A wide variety of ML methodologies have been applied and, despite being applied to the same research problem, this scoping review has not identified a single 'best' method. Two issues arose in studies that compared different ML approaches on the same datasets. First, although different models had similar AUCs during training, not all models generalised well to validation datasets; robust model validation is therefore important to ensure model validity (see below). Second, although in general it is assumed that larger amounts of training data improve model performance, ${ }^{54}$ Richter and Khoshgoftaar ${ }^{41}$ found that equivalent or even better model performance was achievable using reduced datasets (hundreds of thousands vs millions of datapoints). This might be due to high levels of homogeneity in the 'no cancer' class, resulting in fewer instances being required to produce a generalisable model, or as a result of overfitting. Although the requirement for less data for the cancer prediction problem could make ML techniques more accessible to researchers without extensive computing infrastructure and allow smaller datasets to be leveraged for model construction, ML requires over 10 times the amount of data per variable for stable discrimination compared with traditional approaches such as LR. ${ }^{54}$ Instead of regarding data requirements as 'too high' or 'too low', it might be better to consider how much data is required for a particular predictive context. Riley et $a l^{\tilde{5}}$ recently provided an implementation of how to calculate the sample size required to develop specific clinical prediction models, which will help researchers prospectively plan their in silico experiments and avoid using datasets that are too small for the total number of participants or outcome events.

\section{Model validation, comparisons and performance evaluation}

With the exception of the two studies evaluating a previously defined algorithm for colorectal cancer, no other study used external validation datasets to assess model generalisability, instead opting for either a single holdout validation sample or fivefold cross-validation. While useful for assessing overfitting, ${ }^{56}$ these approaches do not account for population bias in the training dataset nor differences in other target populations. Studies seeking to develop ML models should seek to validate models in independent populations, recognising that an advantage of an 'ungeneralisable' model might be insights into cancer risk in other populations. Furthermore, since physicians may code diseases in EHRs differently over time (for instance, due to altered management or incentives), even initially generalisable models may need revalidation over time. ${ }^{23} 57$

Discrimination (ie, the ability to distinguish a patient with a high(er) risk of developing cancer from one with a low(er) risk of developing cancer) was measured in every study using the AUC, as is common in the field. However, discrimination is not the only metric of model performance. ${ }^{58}$ Another important measure of model performance, particularly for the clinical setting, is calibration, that is, establishing that the risk estimates are accurate. ${ }^{59}$ In this setting, this means that the model should not unduly overestimate or underestimate the risk that a patient will develop cancer; to do so would mean that a patient might be subjected to investigations and the associated worry of their likelihood of developing cancer (overestimated risk), or, conversely, underinvestigated and falsely reassured in the case of underestimated risk. Therefore, a highly discriminatory but poorly calibrated model is likely to have poor clinical utility.

None of the studies reviewed here performed calibration analysis, which is not uncommon in this field. Indeed, in their systematic review of 71 studies using ML for clinical prediction for a wide variety of clinical purposes, Christodoulou et al reported that $79 \%$ of studies failed to address the calibration problem. ${ }^{37}$ Therefore, caution must be applied when interpreting and comparing the performance of current ML models based on AUC alone, since is an incomplete measure of performance that must be considered together with methodological aspects such overfitting, measurement error, and population heterogeneity that might influence the estimation of predictive performance. ${ }^{3759}$

\section{Implications for clinical practice}

The ML models described in this scoping review generally show high AUC values. So, are any of these models ready for clinical use? The ColonFlag model ${ }^{39} 43$ is an example has recently been implemented at Barts Health NHS Trust ${ }^{60}$ to identify patients at particularly high risk of CRC, particularly as clinicians struggle to prioritise patients in the backlog created by the Coronaviruses (COVID-19) pandemic. The ColonFlag model is the 
only model identified in this scoping review that has undergone extensive external validation in independent datasets.

New ML models need to be contextualised with currently available best clinical practice in order to fully evaluate their potential clinical value. Comparing the relatively poor AUC values of the Stark $e t a l^{44}$ models with BCRAT revealed that they in fact outperformed it in many cases. In their comparison of their ANN with screening methods for lung cancer such as low-dose CT, chest X-ray and sputum cytology, Hart $e t a l^{8}$ noted that (according to sensitivity and specificity) it outperformed most of the other available non-invasive methods. Thorough side-byside comparisons of newly developed models with other prediction tools would be helpful in establishing future clinical utility.

Finally, this scoping review highlights that model performance should not be evaluated solely on the basis of AUC values but also in terms of other importance performance metrics such as calibration, without which a model might inaccurately assess risk and therefore prompt inappropriate management.

\section{Unanswered questions and future research}

The few models that are currently available are methodologically diverse, rarely validated in independent datasets to ensure generalisability, and do not cover all cancer types. Even if ML techniques offer only small improvements in cancer detection rates, these improvements are likely to be of high clinical significance given the large size of the global population with or at high risk of cancer and the high mortality and costs associated with late cancer diagnoses.

However, the scoping review identifies a number of research gaps that will need to be addressed in order to deliver validated ML-based models to assist clinical decision making. First, future studies must take steps to establish model generalisability through validation in independent cohorts, including those from LMICs. Although the latter may be challenging, it could be argued that even negative generalisability studies might provide an opportunity to learn more about cancer risk factors in different populations. Second, the scoping review fails to establish which ML approach best suits the cancer prediction problem but does show that, where possible, side-by-side comparisons of different methods can reveal important information about generalisability as well as performance and that these comparisons are desirable whenever possible. Third, many important cancer types, particularly 'silent killers' like ovarian cancer, have currently not been the subject of ML modelling approaches; ML could provide an important, low-cost, non-invasive method to identify individuals at high risk of clinically silent cancers that require closer monitoring. Fourth, progress has been made in defining approaches to tailor sample sizes to the specific setting of interest to minimise overfitting and targeting precise estimates of key parameters, and these principles must be applied when testing and validating models to ensure robust model performance. Finally, ML models need to be compared with the best available clinical tools so that their potential clinical utility is transparent.

\section{Limitations of this study}

Our study has a number of limitations. First, despite recognising the need for a scoping review due to the paucity of literature on the topic, we were only able to identify ten papers meeting the inclusion criteria. It is therefore difficult to draw definitive conclusions about the performance of these models. Furthermore, although AUC values provide an indication of how discriminative the models are, they do not allow for meaningful comparisons of models trained and evaluated on different datasets. Six out of ten studies were defined as poor quality due to a lack of controlling for confounders in the study design, which may have introduced significant bias. Finally, we only search the PubMed database and articles published in English, so some papers in other languages or in databases for non-medical disciplines may have been missed.

\section{CONCLUSIONS}

This scoping review highlights that applying ML to cancer prediction is a promising field provided that the identified issues such as generalisability, validation and clinical applicability, model calibration and dataset selection are addressed in future studies. We hope that the identified research gaps focus future research efforts to deliver validated ML-based models to assist and improve clinical decision making

\section{Twitter Holger Kunz @https://orcid.org/0000-0002-2229-4500}

Contributors AAA defined the research question of the scoping review, conducted the literature search and summarised the findings. HK and AGL supervised the research. All authors drafted and revised the manuscript.

Funding AGL is supported by funding from the Wellcome Trust (204841/Z/16/Z), National Institute for Health Research (NIHR) University College London Hospitals Biomedical Research Centre (BRC714/HI/RW/101440), NIHR Great Ormond Street Hospital Biomedical Research Centre (19RX02).

Competing interests None declared.

Patient and public involvement Patients and/or the public were not involved in the design, or conduct, or reporting, or dissemination plans of this research.

Patient consent for publication Not required.

Ethics approval Not applicable. This study does not involve human participants nor animal subjects.

Provenance and peer review Not commissioned; externally peer reviewed.

Data availability statement Data are available in a public, open access repository. Only public published papers were used. No confidential data.

Open access This is an open access article distributed in accordance with the Creative Commons Attribution 4.0 Unported (CC BY 4.0) license, which permits others to copy, redistribute, remix, transform and build upon this work for any purpose, provided the original work is properly cited, a link to the licence is given, and indication of whether changes were made. See: https://creativecommons.org/ licenses/by/4.0/.

\section{ORCID iDs}

Asma Abdullah Alfayez http://orcid.org/0000-0002-7448-2095

Holger Kunz http://orcid.org/0000-0002-2229-4500

Alvina Grace Lai http://orcid.org/0000-0001-8960-8095 


\section{REFERENCES}

1 Siegel RL, Miller KD, Jemal A. Cancer statistics, 2019. CA Cancer J Clin 2019;69:7-34

2 Cancer Research UK. Cancer statistics for the UK, 2020. Available: https://www.cancerresearchuk.org/health-professional/cancerstatistics-for-the-uk

3 Cancer Research UK. Why are cancer rates increasing? 2014. Available: https://scienceblog.cancerresearchuk.org/2015/02/04/ why-are-cancer-rates-increasing/

4 World Health Organization. Early detection of cancer, 2016. Available: https://www.who.int/cancer/detection/en/

5 World Health Organization. Early cancer diagnosis saves lives, cuts treatment costs, 2017. Available: https://www.who.int/newsroom/detail/03-02-2017-early-cancer-diagnosis-saves-lives-cutstreatment-costs

6 Phallen J, Sausen M, Adleff V, et al. Direct detection of early-stage cancers using circulating tumor DNA. Sci Trans/ Med 2017;9. doi:10.1126/scitranslmed.aan2415. [Epub ahead of print: 16 Aug 2017].

7 Blumen $\mathrm{H}$, Fitch K, Polkus V. Comparison of treatment costs for breast cancer, by tumor stage and type of service. Am Health Drug Benefits 2016;9:23-32.

8 Kakushadze Z, Raghubanshi R, Yu W. Estimating cost savings from early cancer diagnosis. Data 2017;2:30.

9 Cancer Research UK. Saving lives, averting costs, 2014. Available: https://www.cancerresearchuk.org/sites/default/files/saving_lives_ averting_costs.pdf

10 Bannister N, Broggio J. Cancer survival by stage at diagnosis for England (experimental statistics): adults diagnosed 2012, 2013 and 2014 and followed up to 2015. Produced in collaboration with Public Health England 2016.

11 Canary Foundation. Early detection facts and figures early detection works. California: Canary Foundation, 2019.

12 Cancer Research UK. Why is early diagnosis important? 2018. Available: https://www.cancerresearchuk.org/about-cancer/cancersymptoms/why-is-early-diagnosis-important

13 Weller DP, Campbell C. Uptake in cancer screening programmes: a priority in cancer control. Br J Cancer 2009;101 Suppl 2:S55-9.

14 Cancer Research UK. About cancer screeing, 2020. Available: https://www.cancerresearchuk.org/about-cancer/screening

15 Smith RA, Andrews KS, Brooks D, et al. Cancer screening in the United States, 2019: a review of current American cancer Society guidelines and current issues in cancer screening. CA Cancer J Clin 2019;69:184-210.

16 Joseph DA, King JB, Dowling NF, et al. Vital signs: colorectal cancer screening test use - United States, 2018. MMWR Morb Mortal Wkly Rep 2020;69:253-9.

17 Power E, Wardle J. Change in public awareness of symptoms and perceived barriers to seeing a doctor following be clear on cancer campaigns in England. Br J Cancer 2015;112 Suppl 1:S22-6.

18 Smith LK, Pope C, Botha JL. Patients' help-seeking experiences and delay in cancer presentation: a qualitative synthesis. Lancet 2005;366:825-31.

19 Balasooriya-Smeekens C, Walter FM, Scott S. The role of emotions in time to presentation for symptoms suggestive of cancer: a systematic literature review of quantitative studies. Psychooncology 2015;24:1594-604

20 Taber JM, Leyva B, Persoskie A. Why do people avoid medical care? A qualitative study using national data. J Gen Intern Med 2015;30:290-7

21 American Cancer Society. Lifetime risk of developing or dying from cancer. Atlanta: American Cancer Society, 2014.

22 Goldstein BA, Navar AM, Pencina MJ, et al. Opportunities and challenges in developing risk prediction models with electronic health records data: a systematic review. J Am Med Inform Assoc 2017;24:198-208.

23 Rose S. Machine learning for prediction in electronic health data. JAMA Netw Open 2018;1:e181404.

24 Rajkomar A, Dean J, Kohane I. Machine learning in medicine. N Engl J Med 2019;380:1347-58.

25 Ye C, Fu T, Hao S, et al. Prediction of incident hypertension within the next year: prospective study using statewide electronic health records and machine learning. J Med Internet Res 2018;20:e22.

26 Hao S, Fu T, Wu Q, et al. Estimating one-year risk of incident chronic kidney disease: retrospective development and validation study using electronic medical record data from the state of Maine. JMIR Med Inform 2017;5:e21.

27 Martín Noguerol T, Paulano-Godino F, Martín-Valdivia MT, et al. Strengths, weaknesses, opportunities, and threats analysis of artificial intelligence and machine learning applications in radiology. $J$ Am Coll Radiol 2019;16:1239-47.
28 Bera K, Schalper KA, Rimm DL, et al. Artificial intelligence in digital pathology - new tools for diagnosis and precision oncology. Nat Rev Clin Oncol 2019;16:703-15.

29 Hastie T, Tibshirani R, Friedman J. The elements of statistical learning: data mining, inference, and prediction. Berlin: Springer Science \& Business Media, 2009.

30 Kourou K, Exarchos TP, Exarchos KP, et al. Machine learning applications in cancer prognosis and prediction. Comput Struct Biotechnol J 2015;13:8-17.

31 Richter AN, Khoshgoftaar TM. A review of statistical and machine learning methods for modeling cancer risk using structured clinical data. Artif Intell Med 2018;90:1-14.

32 Munn Z, Peters MDJ, Stern C, et al. Systematic review or scoping review? guidance for authors when choosing between a systematic or scoping review approach. BMC Med Res Methodol 2018;18:143.

33 Peters MDJ. In no uncertain terms: the importance of a defined objective in scoping reviews. JBI Database System Rev Implement Rep 2016;14:1-4.

34 Beam AL, Kohane IS. Big data and machine learning in health care. JAMA 2018;319:1317-8.

35 Stang A. Critical evaluation of the Newcastle-Ottawa scale for the assessment of the quality of nonrandomized studies in metaanalyses. Eur J Epidemiol 2010;25:603-5.

36 Waegeman W, De Baets B, Boullart L. Roc analysis in ordinal regression learning. Pattern Recognit Lett 2008;29:1-9.

37 Christodoulou E, Ma J, Collins GS, et al. A systematic review shows no performance benefit of machine learning over logistic regression for clinical prediction models. J Clin Epidemiol 2019;110:12-22.

38 Hart GR, Roffman DA, Decker R, et al. A multi-parameterized artificial neural network for lung cancer risk prediction. PLoS One 2018;13:e0205264.

39 Hornbrook MC, Goshen R, Choman E, et al. Early colorectal cancer detected by machine learning model using gender, age, and complete blood count data. Dig Dis Sci 2017;62:2719-27.

40 Miotto R, Li L, Kidd BA, et al. Deep patient: an unsupervised representation to predict the future of patients from the electronic health records. Sci Rep 2016;6:26094.

41 Richter AN, Khoshgoftaar TM. Efficient learning from big data for cancer risk modeling: a case study with melanoma. Comput Biol Med 2019;110:29-39.

42 Roffman D, Hart G, Girardi M, et al. Predicting non-melanoma skin cancer via a multi-parameterized artificial neural network. Sci Rep 2018;8:1701.

43 Schneider JL, Layefsky E, Udaltsova N, et al. Validation of an algorithm to identify patients at risk for colorectal cancer based on laboratory test and demographic data in diverse, community-based population. Clin Gastroenterol Hepatol 2020;18:2734-41.

44 Stark GF, Hart GR, Nartowt BJ, et al. Predicting breast cancer risk using personal health data and machine learning models. PLoS One 2019;14:e0226765

45 Wang $\mathrm{H}-\mathrm{H}$, Wang $\mathrm{Y}-\mathrm{H}$, Liang $\mathrm{C}-\mathrm{W}$, et al. Assessment of deep learning using Nonimaging information and sequential medical records to develop a prediction model for nonmelanoma skin cancer. JAMA Dermatol 2019;155:1277.

46 Wang Y-H, Nguyen P-A, Islam MM, et al. Development of deep learning algorithm for detection of colorectal cancer in EHR data. Stud Health Technol Inform 2019;264:438-41.

47 Zhao D, Weng C. Combining PubMed knowledge and EHR data to develop a weighted Bayesian network for pancreatic cancer prediction. J Biomed Inform 2011;44:859-68.

48 Gail MH, Brinton LA, Byar DP, et al. Projecting individualized probabilities of developing breast cancer for white females who are being examined annually. J Natl Cancer Inst 1989;81:1879-86.

49 Lorena AC, Jacintho LFO, Siqueira MF, et al. Comparing machine learning classifiers in potential distribution modelling. Expert Syst Appl 2011;38:5268-75.

50 Usher-Smith JA, Walter FM, Emery JD, et al. Risk prediction models for colorectal cancer: a systematic review. Cancer Prev Res 2016;9:13-26.

51 Gray EP, Teare MD, Stevens J, et al. Risk prediction models for lung cancer: a systematic review. Clin Lung Cancer 2016;17:95-106.

52 Usher-Smith JA, Emery J, Kassianos AP, et al. Risk prediction models for melanoma: a systematic review. Cancer Epidemiol Biomarkers Prev 2014;23:1450-63.

53 Rastogi T, Hildesheim A, Sinha R. Opportunities for cancer epidemiology in developing countries. Nat Rev Cancer 2004;4:909-17.

54 van der Ploeg T, Austin PC, Steyerberg EW. Modern modelling techniques are data hungry: a simulation study for predicting dichotomous endpoints. BMC Med Res Methodol 2014;14:137. 
55 Riley RD, Ensor J, Snell KIE, et al. Calculating the sample size required for developing a clinical prediction model. $B M J$ 2020;368:m441.

56 Kohavi R. A study of cross-validation and bootstrap for accuracy estimation and model selection. Inljcai 1995;14:1137-45.

57 Bergquist SL, Brooks GA, Keating NL, et al. Classifying lung cancer severity with ensemble machine learning in health care claims data. Proc Mach Learn Res 2017;68:25-38.

58 Steyerberg EW, Vickers AJ, Cook NR, et al. Assessing the performance of prediction models: a framework for traditional and novel measures. Epidemiology 2010;21:128-38.
59 Van Calster B, McLernon DJ, van Smeden M, et al. Calibration: the Achilles heel of predictive analytics. BMC Med 2019;17:230.

60 Downing M. Barts health using Al to prioritise care for colon cancer patients, 2020. Available: https://www.bartshealth.nhs.uk/news/ barts-health-using-ai-to-prioritise-care-for-high-risk-colon-cancerpatients-8867

61 Kinar Y, Kalkstein N, Akiva P, et al. Development and validation of a predictive model for detection of colorectal cancer in primary care by analysis of complete blood counts: a binational retrospective study. $J$ Am Med Inform Assoc 2016;23:879-90. 